\title{
Bridging the gap between ab initio simulations and experiments through EIS
}

\section{Mohit Mehta $^{a, b}$, Vamsci Venkat Bevara ${ }^{a, b}$, and Petru Andreia,b}

aDepartment of Electrical and Computer Engineering, Florida State University, Tallahassee, FL 32310, USA

${ }^{b}$ Aeropropulsion, Mechatronics and Energy, Tallahassee, FL 32310, USA

\section{Introduction}

We have developed an analytical solution to compute the impedance spectra using ab initio parameters such as total Gibbs free energy change for the electron transfer electrode. The impedance model is developed to relate DFT computed parameters to experiments using Electrochemical Impedance Spectroscopy (EIS) using the analytical solution of the Marcus-Hush-Chidsey (MHC) reaction rate theory developed by Zeng et al. [1]. Next, we compare the reaction rates computed by Butler-Volmer and $\mathrm{MHC}$ theory and impedance responses using the two reaction models. Finally,
we provide a few analytical predictions of our analytical model using the MHC theory.

\section{Marcus-Hush-Chidsey kinetics}

The analytical MHC theory is introduced and some DFT techniques are outlined to provide different parameters to calculate the reaction rate

The reaction rate constant using MHC kinetics is computed using an analytical equation given by Zeng et al.
$k_{\mathrm{MHC}}=\frac{k_{0} a}{k\left(\frac{\lambda}{k_{B} T}, 0\right)}\left[k\left(\frac{\lambda}{k_{B} T}, \frac{-\eta}{V_{T}}\right)-k\left(\frac{\lambda}{k_{B} T}, \frac{\eta}{V_{T}}\right)\right]$

where

$k\left(\lambda^{\prime}, \eta^{\prime}\right) \approx \frac{\sqrt{\lambda^{\prime}}}{1+e^{\left(-\eta^{\prime}\right)}} \operatorname{efrc}\left(\frac{\lambda^{\prime}-\sqrt{1+\sqrt{\lambda^{\prime}}+\eta^{\prime 2}}}{2 \sqrt{\lambda^{\prime}}}\right)$

The reorganization energies $\lambda$ needs to be calculated using DFT - dielectric properties of the solvent

solvation energy

electronic coupling energy

Activation energy on reductio

\section{$\stackrel{\text { lonic radius }}{\text { Oxygen mole }}$ \\ 02021123000 \\ $001,\{000\}$ atoms}

Figure showing configuration of model system for calculation

of the reorganizalion energy using DFT (from Rel. 2.)

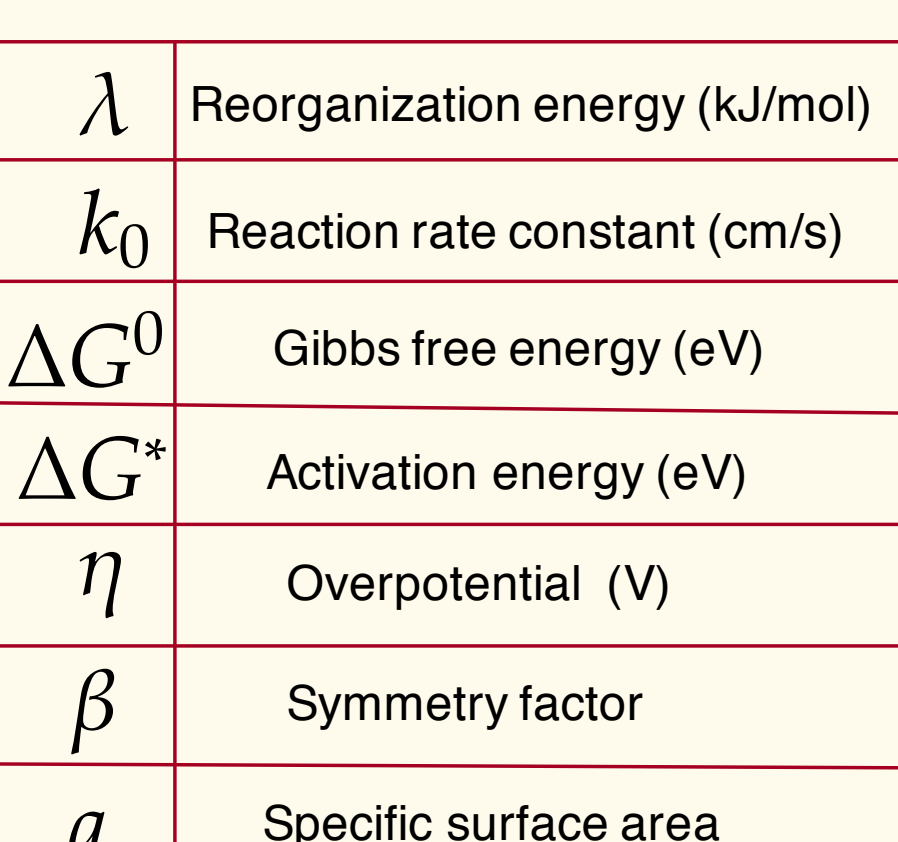

$k_{0}$ Reacioin rate constant (cm/s) $\Delta G^{*}$ Adtivation energy (ev) Symmety factor

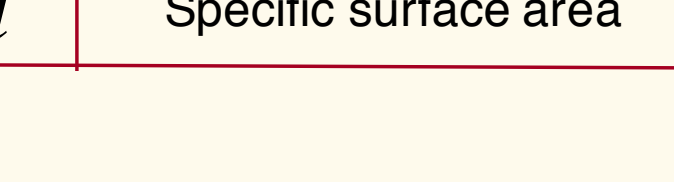

\footnotetext{
This energy can be calculated using the Born energy of sol
\[ \lambda_{\text {out }}=\frac{e^{2}}{8 \pi \epsilon_{0} k_{B} T}\left(\frac{1}{a_{0}}-\frac{1}{2 d}\right)\left(\frac{1}{\epsilon_{o p}}-\frac{1}{\epsilon_{s}}\right) \]
}

$\lambda_{\text {inner }}=\left[E\left(D^{+} \mid D\right)-E\left(D^{+} \mid D^{+}\right)\right]+\left[E\left(A^{-} \mid A\right)-E\left(A^{-} \mid A^{-}\right)\right]$

Total reorganization energy

Comparison between the reaction rates in MHC and BV

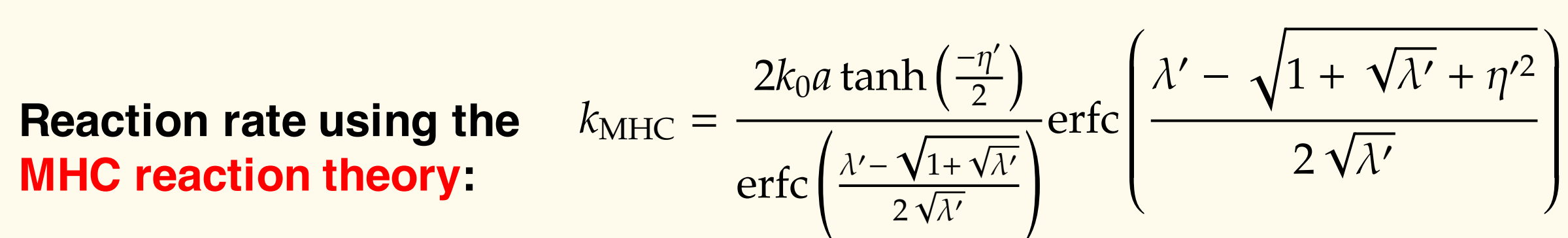

$\begin{aligned} & \text { Reaction rate using the } \\ & \mathrm{BV} \text { reaction theory }[3]:\end{aligned} k^{\mathrm{BV}(\beta=0.5)}=2 k_{0} a \sinh \left(\frac{n}{2 V_{T}} \eta\right)$
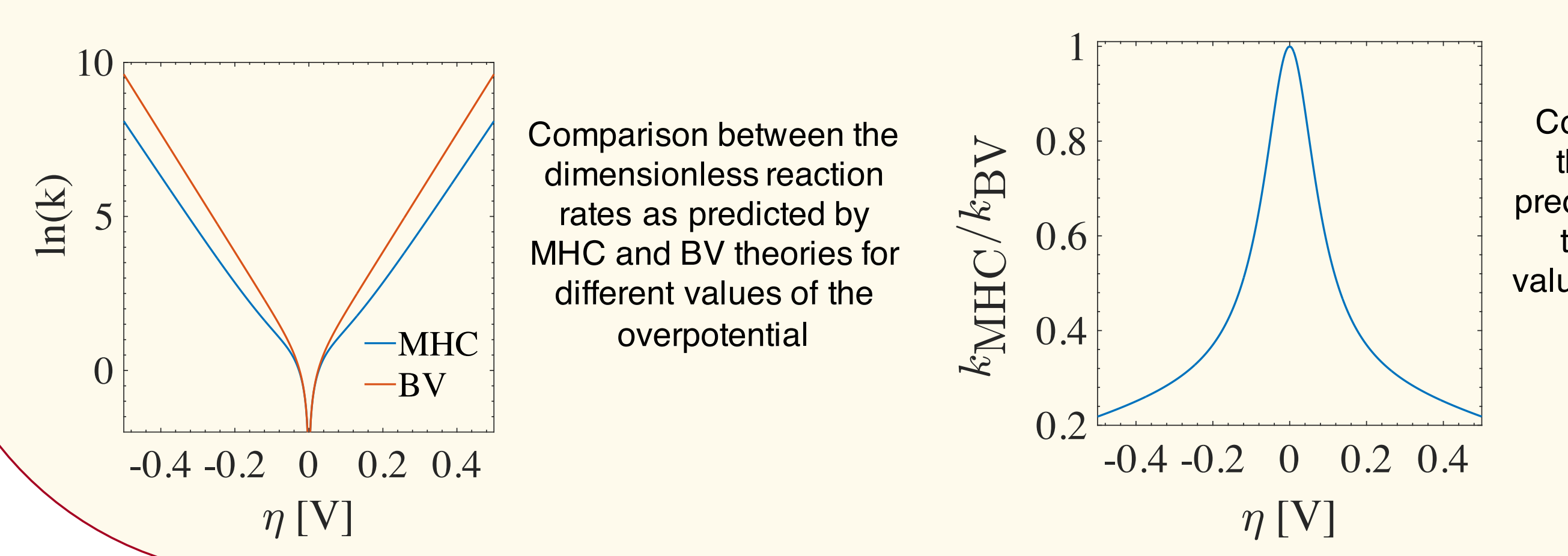

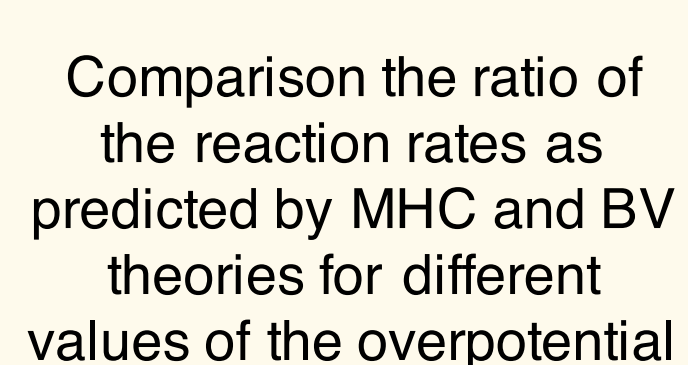

Reaction coordinate $\longrightarrow$ Energy diagram for Marcus

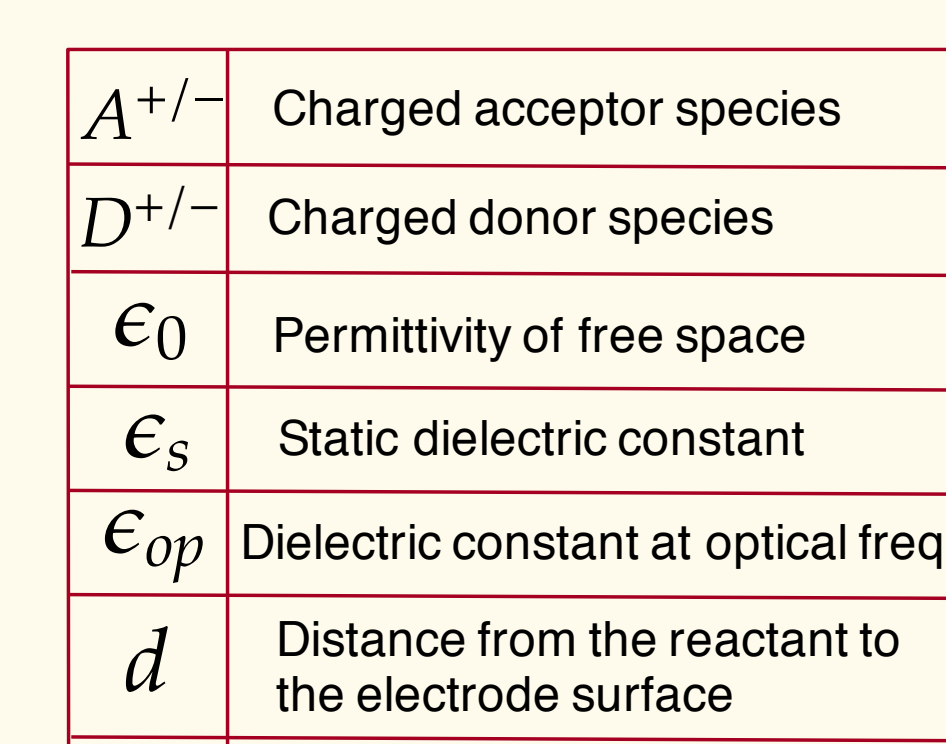

\begin{tabular}{|l|l|}
\hline$d$ & $\begin{array}{l}\text { Distance from the reactant } \\
\text { the electrode surface }\end{array}$ \\
\hline &
\end{tabular}
Bond reorganization energy
This energy can be calculated using Nelson's four point method $[2]$

\section{Impedance spectra modeling} Air Batteries. Hence,
the following diffusion equation $[3]$

$$
\frac{\partial\left(\epsilon c_{0_{2}}\right)}{\partial t}=\frac{\partial}{\partial}\left(D_{\text {eff }} \frac{\partial c_{0_{2}}}{\partial x}\right)-k_{c} c_{0_{2}}
$$

The reaction rate constant is given by $k_{M H C}$. Under steady state conditions,
the Faradaic equation becomes

$$
I_{F}=n A F k_{\mathrm{MHC}} c_{o_{2}}^{*} \sqrt{\frac{D_{\text {eff }}}{k_{\mathrm{MHC}}}} \tanh \left(\sqrt{\frac{k_{\mathrm{MHC}}}{D_{\text {eff }}}} l\right)
$$

Using small-signal analysis

$v=V+\delta v e^{j \omega t} \quad i=I+\delta i e^{j \omega t} \quad \eta=\eta_{0}+\delta i e^{j \omega t}$

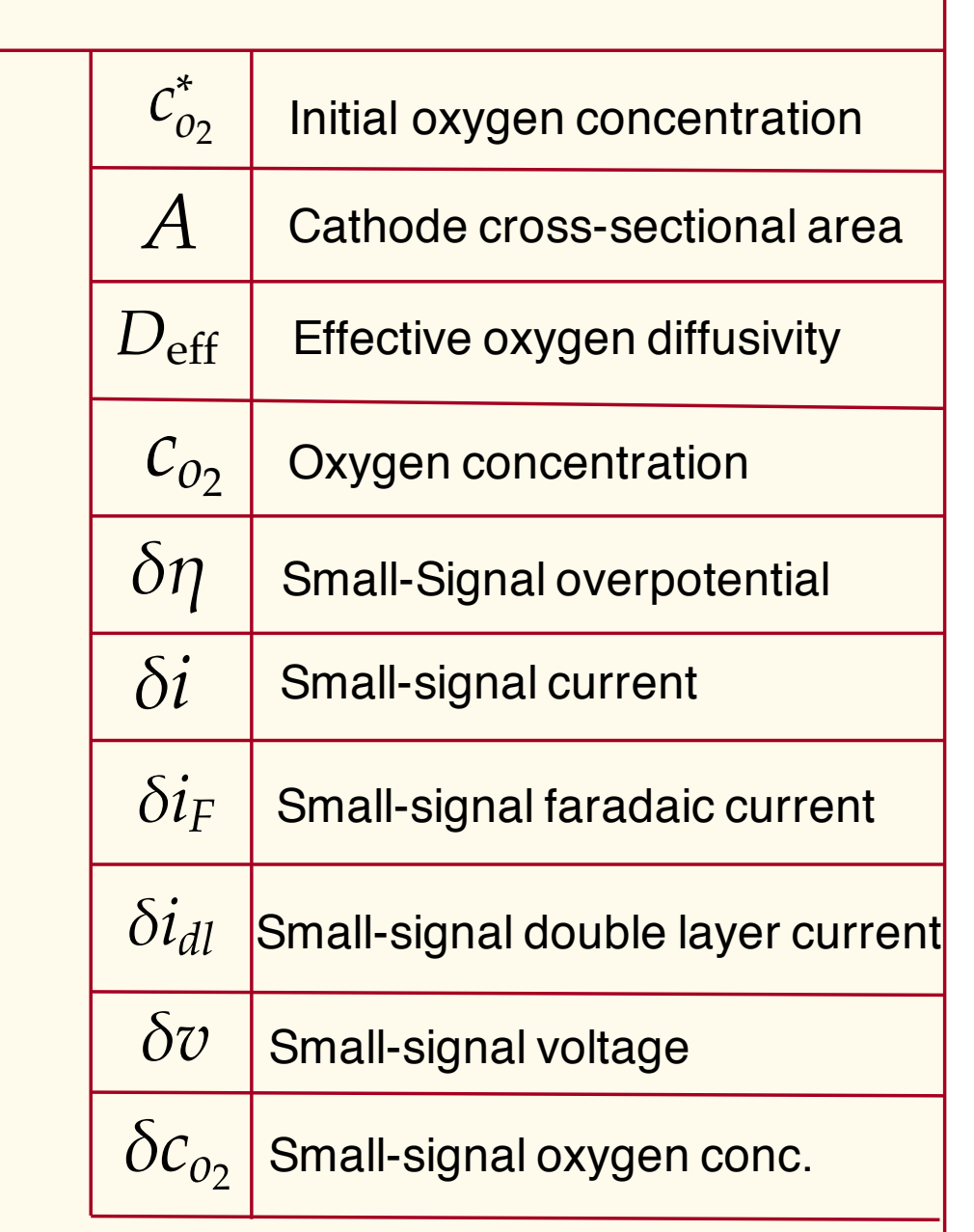

The faradaic impedance is computed using

$Z_{F}=\frac{-\delta \eta}{\delta k} \frac{\delta k}{\delta i_{F}} \quad$ where, $\quad \delta i_{F}=n F A k_{\mathrm{MHC}} \int_{0}^{L_{c}} \delta c_{0_{2}} d x+n F A \frac{\partial k}{\partial \eta} \delta \eta \int_{0}^{L_{c}} c_{0_{2}} d x$

and $\frac{-\delta \eta}{\delta k}$ is computed by linearizing $k_{\mathrm{MHC}}=\frac{k_{0} a}{k\left(\frac{\lambda}{k_{B} T}, 0\right)}\left[k\left(\frac{\lambda}{k_{B} T}, \frac{-\eta}{V_{T}}\right)-k\left(\frac{\lambda}{k_{B} T}, \frac{\eta}{V_{T}}\right)\right]$

\section{Impedance spectra comparison}

Comparison between two charge transfer kinetics formalisms: Butler-Volmer and Marcus-Hush-Chidsey

Impedance equation using Butler-Volmer reaction theory

$$
Z_{F}^{\mathrm{BV}(\beta=0.5)}(\omega)=\frac{Z_{0} f\left(\frac{\eta}{V_{T}}\right)}{\left[1-\frac{k_{\mathrm{BV}}}{j \omega \epsilon}+\frac{k_{\mathrm{BV}} \sqrt{k_{\mathrm{BV}}} \tanh \left(\sqrt{\frac{k_{\mathrm{BV}}+j \omega \epsilon}{D_{\mathrm{eff}}} l}\right)}{j \omega \epsilon \sqrt{k_{\mathrm{BV}}+j \omega \epsilon} \tanh \left(\sqrt{\frac{k_{\mathrm{BV}}}{D_{\mathrm{eff}}} l}\right)}\right]}
$$

$f\left(\eta^{\prime}\right)=\frac{2 \tanh \left(\frac{-n}{2} \eta^{\prime}\right)}{n} \quad$ and $\quad Z_{0}=\frac{V_{T}}{i_{F}}$

Impedance equation using Marcus-Hush-Chidsey reaction theory

$$
\begin{aligned}
& \mathrm{Z}_{F}^{\mathrm{MHC}}(\omega) \\
& Z_{0} f\left(\frac{\eta}{V_{T}}\right) \\
& \left.1-\frac{k_{\mathrm{MHC}}}{j \omega \epsilon}+\frac{k_{\mathrm{MHC}} \sqrt{k_{\mathrm{MHC}}}}{j \omega \epsilon \sqrt{k_{\mathrm{MHC}}+j \omega \epsilon}} \frac{\tanh \left(\sqrt{\frac{k_{\mathrm{MHC}}+j \omega e}{D_{\text {eff }}}} l\right)}{\tanh \left(\sqrt{\frac{k_{\mathrm{MHC}}}{D_{\text {eff }}}} l\right)}\right]
\end{aligned}
$$

It can be shown that the slow diffusion of the oxygen is responsible for the low power density of $L$ -

where
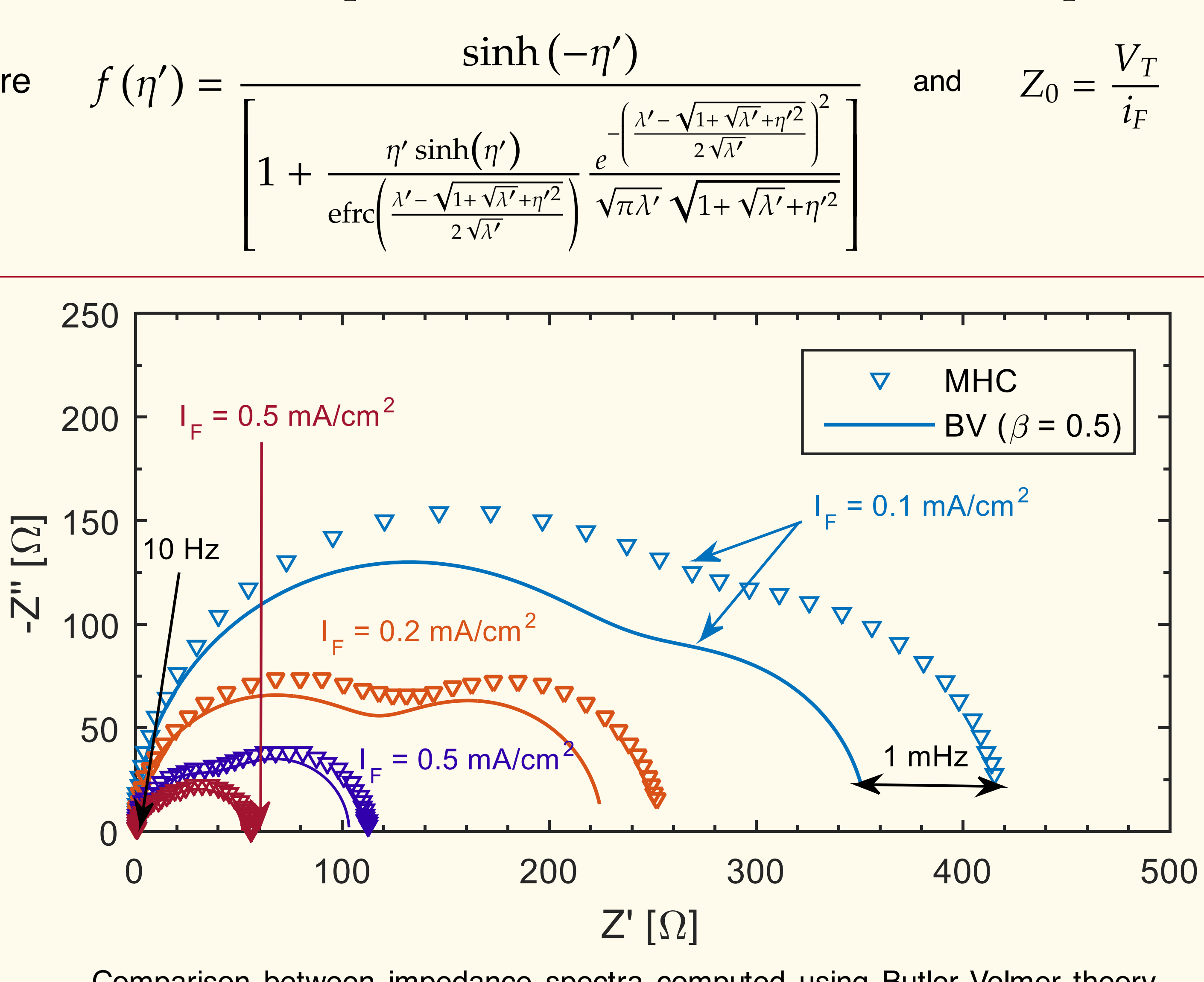

Comparison between impedance spectra computed using
and by using MHC theory for different discharge currents.
Analytical predictions using MHC theory The effect of different physical parameters on the impedance spectra are shown below:

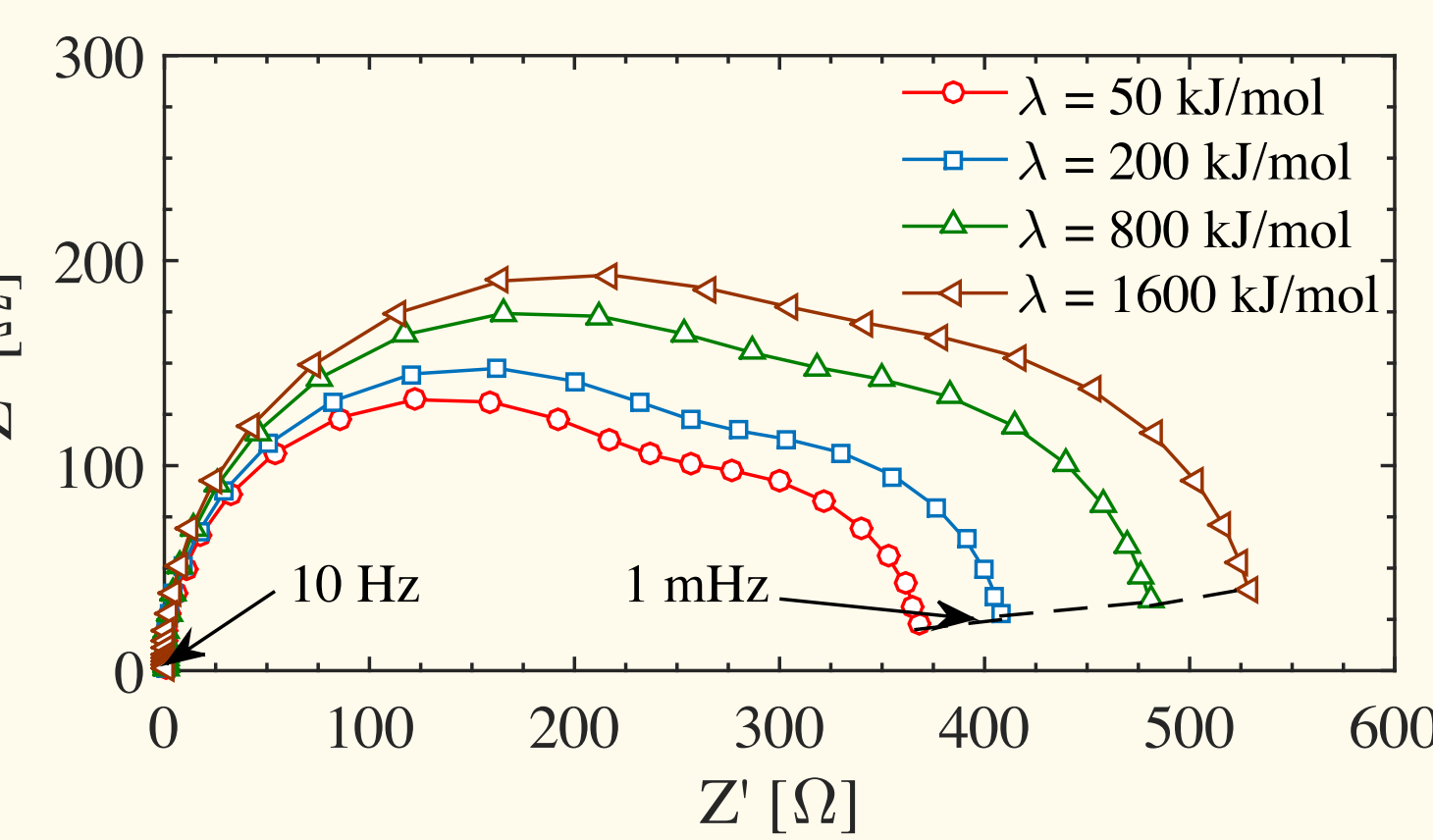

Nyquist plots for different values of total reorganization energy for a $\mathrm{I}_{\text {dis }}=0.1 \mathrm{~mA} / \mathrm{cm}^{2}$

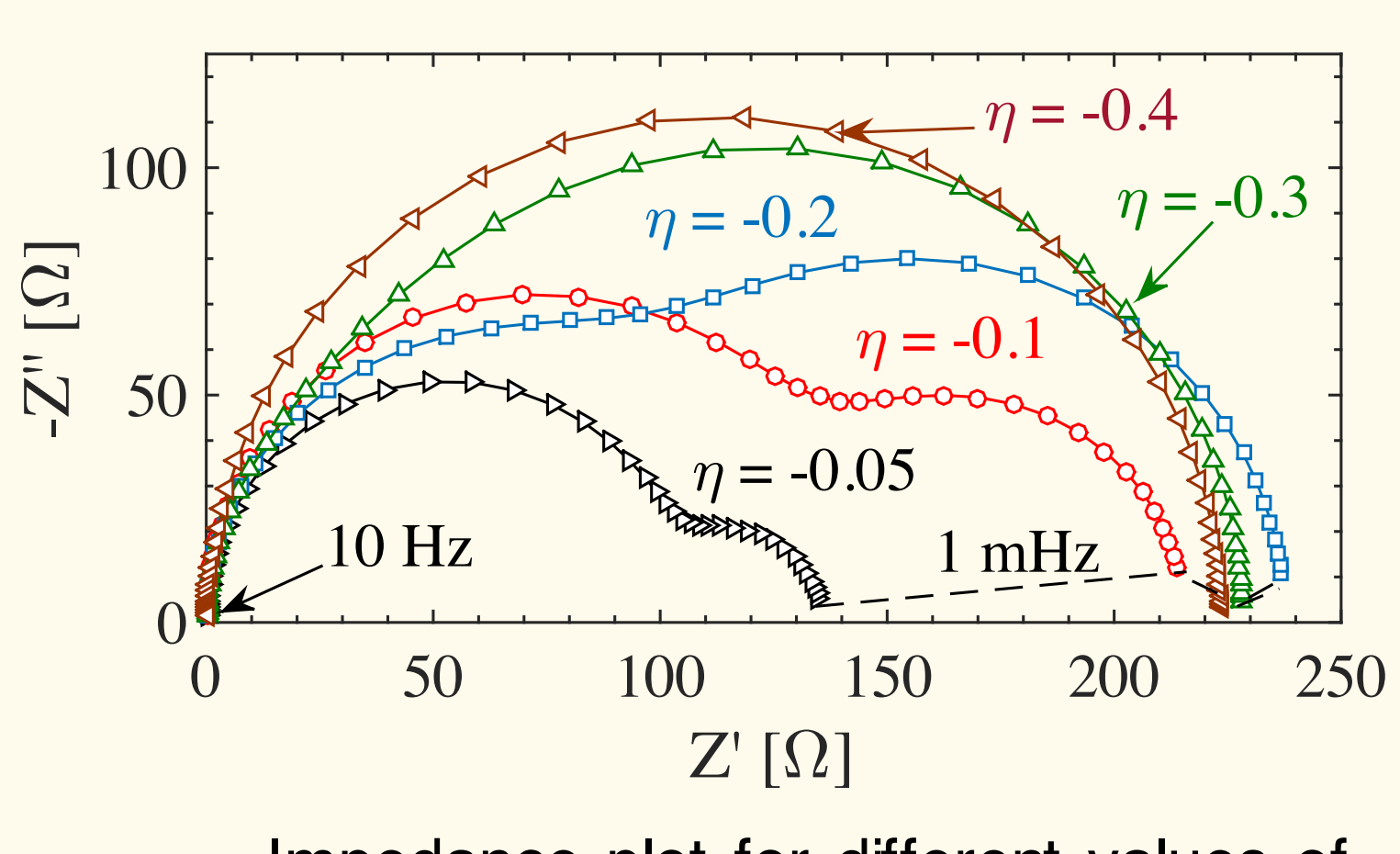

Impedance plo for
overpotential

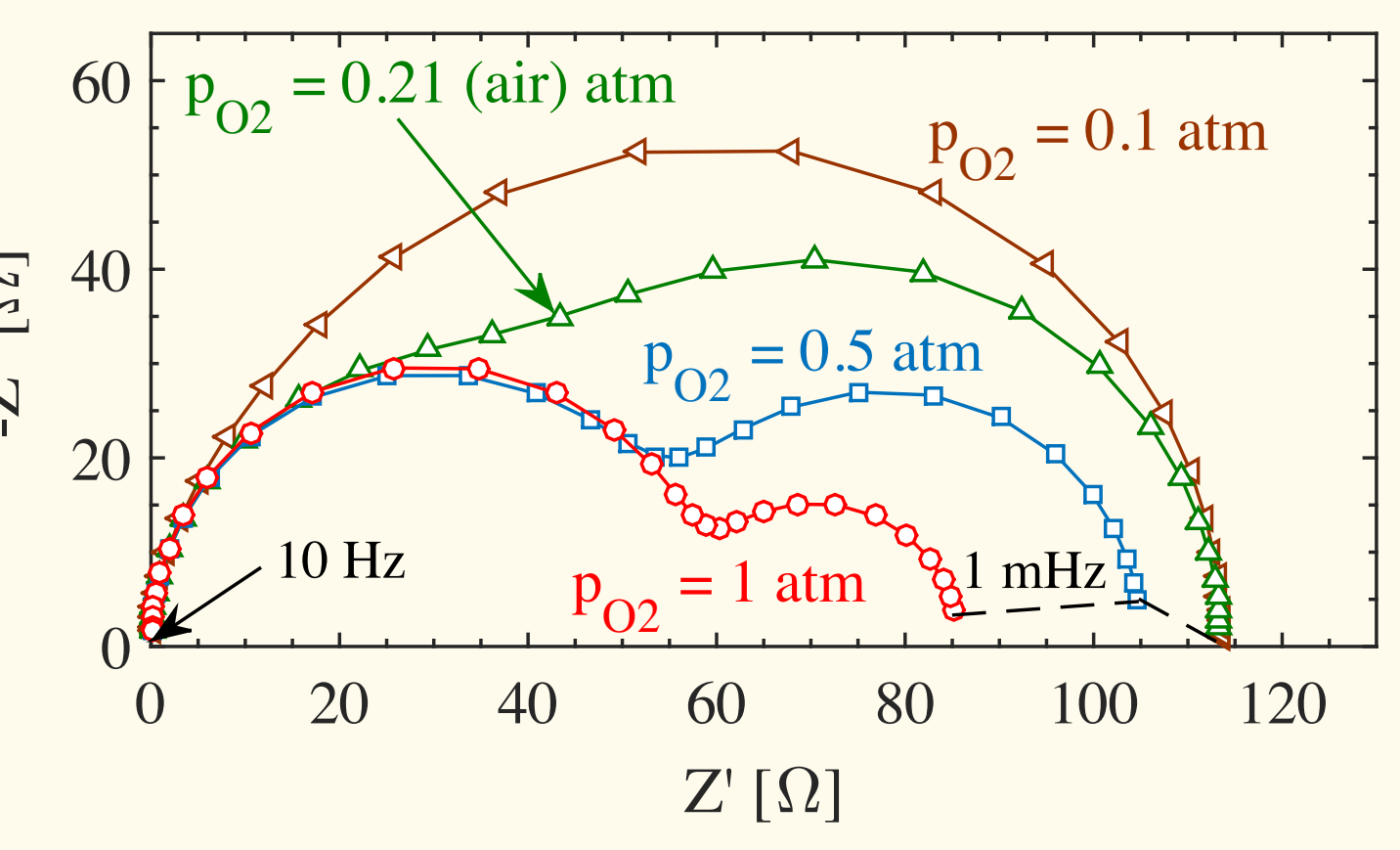

Impedance plots for different values of total
reorganization energy for $\mathrm{I}_{\mathrm{dis}}=0.5 \mathrm{~mA} / \mathrm{cm}^{2}$

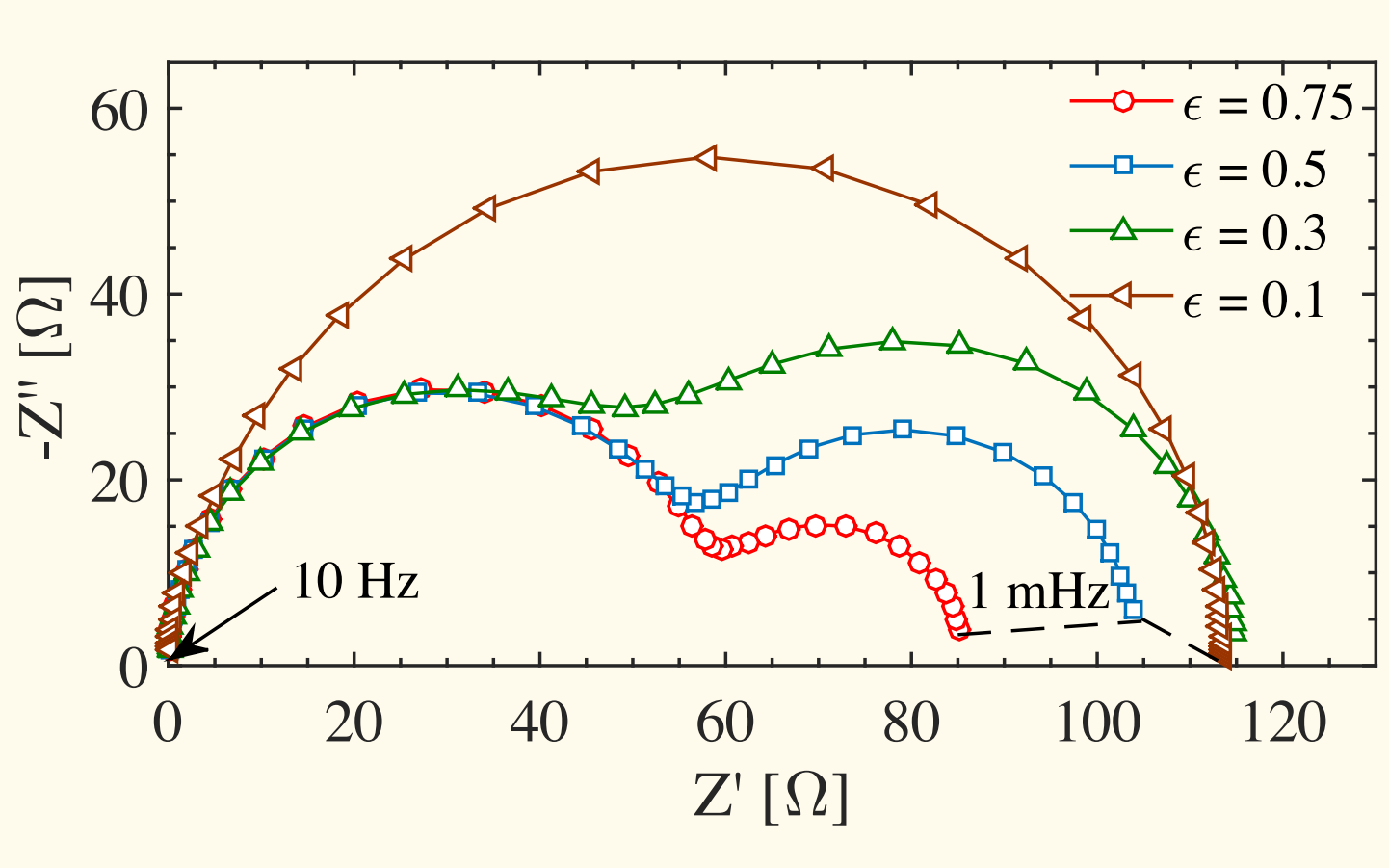

Impedance plots for different values of total

\section{Conclusion}

We have investigated the faradaic impedance of $L$-air batteries with organic electrolyte and derived a closed-form equation for the total electrochemical impedance using Marcus-Hush-Chidsey of the reorganization energy, Gibbs free energy, activation potential, and electronic coupling energy. The reorganization energy as well as the overpotential has profound effect on the total impedance and thus our analytical solution can be used to bridge the gap
experiments using electrochemical impedance spectroscopy.

Our analytical solution could in principle be used to verify existing and new electrode reaction mechanisms obtained by DFT computations. Impedance spectroscopy can provide a simple yet

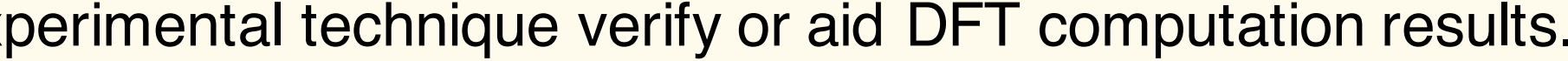

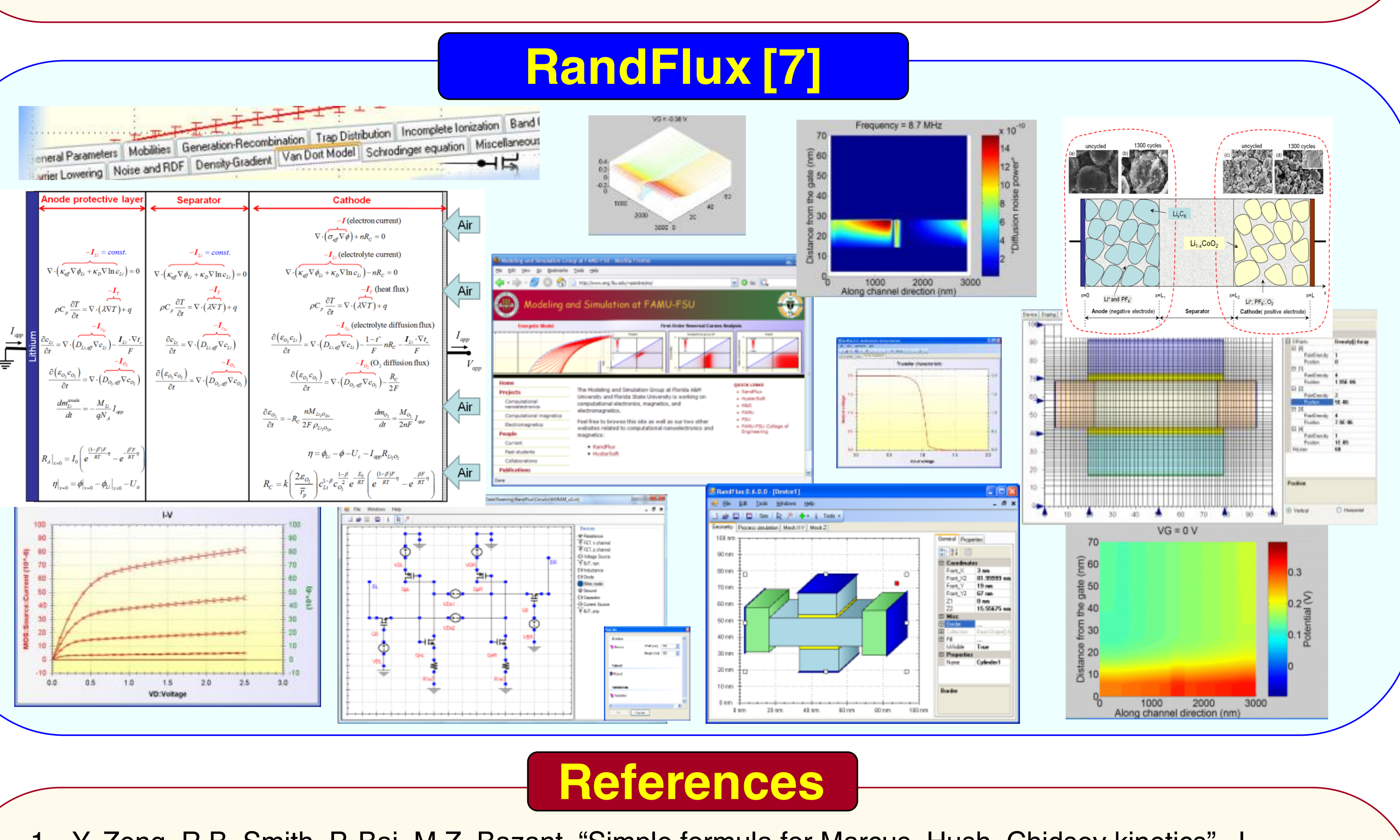

Y. Zeng, R.B. Smith, P. Bai, M.Z. Bazant, "Simple formula for Marcus-Hush-Chidsey kinetics", J.
Electroanal. Chem. 735 (2014) 77-83.

2. S. Kazemiabnavi, P. Dutta, S. Baneriee, "A density functional theory based study of the electron transfer
reaction at the cathode-electrolyte interface in ithium-air batteries", Phys. Chem. Chem. Phys. 17 (2015)
117470-11751. 3. M. Menta, V. Bevara, P. Andrei, "Maximum theoretical
electrolyte", J. Power Sources. 286 (2015) 299-308.

4. P. Andrei, J.P. Zheng, M. Hendrickson. E.J. Plichta, Some Possible Approach
Density of Li-Air Batteries, J. Electrochene. Soc. 157 (2010) A1287-AA295. 5. Mehta, M. Mixon, G., Zheng, J.P. and Andrei, P. 2013 , "Analytical Electrochemical Impedance Modeling
of Li-Air Batteries under D.C. Discharge", J. of the Electrochem. Soc,, vol. 160, no. 11, pp. A2033-A2045. 6. M. Menta, P. Andrei, "Modeling and Simula
Batteries", ECS Trans. 61 (2014) 39-55.

7. RandFFux, User's manual v.0.6, Florida State University, http://www.eng.fsu.edu/ms/RandFlux. 